\title{
Rapid spread of the SARS-CoV-2 Delta variant in some French regions, June 2021
}

Samuel Alizon ${ }^{1}$, Stéphanie Haim-Boukobza ${ }^{2}$, Vincent Foulongne ${ }^{3}$, Laura Verdurme ${ }^{2}$, Sabine Trombert-Paolantoni² , Emmanuel Lecorche $^{2}$, Bénédicte Roquebert ${ }^{2}$, Mircea T. Sofonea ${ }^{1}$

1. MIVEGEC, CNRS, IRD, Université de Montpellier, Montpellier, France

2. Laboratoire Cerba, Saint Ouen L'Aumône, France

3. Laboratoire de Virologie, CHU de Montpellier, France

Correspondence: Samuel Alizon (samuel.alizon@cnrs.fr)

Citation style for this article:

Alizon Samuel, Haim-Boukobza Stéphanie, Foulongne Vincent, Verdurme Laura, Trombert-Paolantoni Sabine, Lecorche Emmanuel, Roquebert Bénédicte, Sofonea Mircea T.. Rapid spread of the SARS-CoV-2 Delta variant in some French regions, June 2021. Euro Surveill. 2021;26(28): pii=2100573. https://doi.org/10.2807/15607917.ES.2021.26.28.2100573

We analysed 9,030 variant-specific RT-PCR tests performed on SARS-CoV-2-positive samples collected in France between 31 May and 21 June 2021. This analysis revealed rapid growth of the Delta variant in three of the 13 metropolitan French regions and estimated a $+79 \%$ (95\% confidence interval: $52-110 \%$ ) transmission advantage compared with the Alpha variant. The next weeks will prove decisive and the magnitude of the estimated transmission advantages of the Delta variant could represent a major challenge for public health authorities.

The evolution of severe acute respiratory syndrome coronavirus 2 (SARS-CoV-2) is characterised by the emergence of several variants which have caused major epidemics in the United Kingdom (UK) [1,2], Brazil [3], and South Africa [4]. In May 2021, the Delta variant (Phylogenetic Assignment of Named Global Outbreak (Pango) lineage designation B.1.617.2), first detected in India was designated a variant of concern by the World Health Organization [5] and was associated with an epidemic rebound in the UK [6]. Analyses showed a transmission advantage of ca $66 \%$ (95\% confidence interval (Cl): $28-113 \%$ ) over the Alpha variant (B.1.1.7) in England [7] and the Delta variant appeared slightly more prone to immune evasion [8].

As evident with the emergence of the Alpha variant earlier in 2021, there appears to be a 2-month shift between the French and epidemics in the UK $[9,10]$. Therefore, it is timely to investigate the potential early spread of the Delta variant in France to devise appropriate public health responses.

\section{Detection of the SARS-CoV-2 Delta variant} We analysed 9,030 RT-PCR variant-specific screening tests (VirSNiP assay, TIB Molbiol, Berlin, Germany) performed on samples collected in France by the CERBA network of medical analysis laboratories in patients from 5 to 80 years old between 31 May and 21 June 2021 that were positive for SARS-CoV-2. The assay screens for the presence of the $\mathrm{E}_{4} 84 \mathrm{~K}$, the $\mathrm{E} 484 \mathrm{Q}$, and the $\mathrm{L} 425 \mathrm{R}$ mutations (Supplement). A $484 \mathrm{~K}+/ 484 \mathrm{Q}-/ 452 \mathrm{R}-$ profile is consistent with the Beta, Gamma, or Eta variants (B.1.351, P.1, and B.1.525 Pango lineages, respectively), whereas a $484 \mathrm{~K}-/ 484 \mathrm{Q}-/ 452 \mathrm{R}+$ profile is consistent with mainly the Delta variant [11]. While other variants of interest (VOI) or under monitoring (VUM) are consistent with the latter profile - e.g. Kappa VOI or $21 \mathrm{C} \mathrm{VUM}$ (B.1.427 and B.1.429 Pango lineages, respectively) sequencing surveys have shown their relative frequency to be negligible in France [12]. To validate VirSNiP assay, samples already characterized by NGS sequencing (COVIDSEQ Illumina) were tested. Of those tested, 333 samples were found with a $484 \mathrm{~K}-/ 484 \mathrm{Q}-/ 452 \mathrm{R}+$ profile, consistent with the Delta variant, in 329 cases (98.8\%). Three cases showed a $452 \mathrm{R}+$ profile with $484 \mathrm{~K}$ or $484 \mathrm{Q}$ mutations uninterpretable (0.9\%) and one case showed a $484 \mathrm{~K}-/ 484 \mathrm{Q}+/ 452 \mathrm{R}+$ profile (0.3\%). Therefore, the specificity of the VirSNiP assay to define $484 \mathrm{~K}-/ 484 \mathrm{Q}-/ 452 \mathrm{R}+$ profile as the Delta variant is $98.8 \%$. Of 342 tests with a $484 \mathrm{~K}-/ 484 \mathrm{Q}-/ 452 \mathrm{R}+\mathrm{VirSNiP}$ profile, the sequencing assay showed 329 Delta variants, two variants B.1.1.7/452R, two variants $20 \mathrm{D}$ and 10 sequences that were uninterpretable because of NGS coverage less than $98.5 \%$. Calculated sensitivity was $96.2 \%$ when including uninterpretable sequences and $99 \%$ when discarding the 10 uninterpretable sequences. These tests were performed on samples from different French regions, with the majority from the Île-de-France (the Paris area; 50\%; 4,483/9,030 tests; Supplementary Figure $\mathrm{S}_{1}$ ) and were mostly from non-hospitalised individuals (93\%; 8,404/9,030 tests; Supplementary Table S1).

\section{Variant-screening test results}

Using a multinomial regression model [13], we found that samples bearing the $\mathrm{L} 452 \mathrm{R}$ mutation but not the E484K or the E484Q mutation (consistent with the Delta 
Factors associated with the detection of potential SARS-CoV-2 variants compared with the Alpha variant, as assessed by relative risk ratios using a multinomial log-linear model, France, 31 May-21 June $2021(n=8,190)$

\begin{tabular}{|c|c|c|c|c|c|c|c|}
\hline & & \multicolumn{6}{|c|}{ RRR per variant } \\
\hline & & \multicolumn{2}{|c|}{ Beta/Gamma/Eta variants ${ }^{\mathrm{a}}$} & \multicolumn{2}{|c|}{ Delta variant ${ }^{\mathrm{a}}$} & \multicolumn{2}{|c|}{ Other variants ${ }^{a}$} \\
\hline & & RRR & $95 \% \mathrm{Cl}$ & RRR & $95 \% \mathrm{Cl}$ & RRR & $95 \% \mathrm{Cl}$ \\
\hline \multicolumn{2}{|l|}{ Age } & 1.07 & $1.00-1.10$ & NS & $0.94-1.20$ & NS & $0.98-1.10$ \\
\hline \multirow{2}{*}{ Sample origin } & Hospital samples & \multicolumn{6}{|c|}{ Ref. } \\
\hline & Non-hospital samples & 0.62 & $0.49-0.80$ & NS & $0.64-1.60$ & NS & $0.71-1.10$ \\
\hline \multirow{6}{*}{$\begin{array}{l}\text { Interaction between the sampling } \\
\text { region and the calendar date of } \\
\text { sampling }\end{array}$} & Normandie & NS & $0.97-1.40$ & 1.72 & $1.30-2.20$ & 1.40 & $1.20-1.60$ \\
\hline & Hauts-de-France & 1.29 & $1.00-1.60$ & 2.00 & $1.50-2.70$ & 1.52 & $1.30-1.80$ \\
\hline & Ile-de-France & 1.19 & $1.10-1.30$ & 2.23 & $1.90-2.60$ & 1.50 & $1.40-1.60$ \\
\hline & Provence-Alpes-Côte d'Azur & NS & $0.75-1.30$ & 1.92 & $1.40-2.70$ & NS & $0.92-1.30$ \\
\hline & Centre-Val-de-Loire & NS & $0.56-1.20$ & NS & $0.51-1.70$ & 1.29 & $1.00-1.70$ \\
\hline & Other regions & NS & $0.71-1.00$ & NS & $0.70-1.30$ & 1.40 & $1.20-1.60$ \\
\hline
\end{tabular}

$\mathrm{Cl}$ : confidence interval; NS: non-significant; RRR: relative risk ratio; SARS-CoV-2: severe acute respiratory syndrome coronavirus 2 ; VOI: variant of interest; VUM: variant under monitoring.

a A $484 \mathrm{~K}+/ 484 \mathrm{Q}-/ 452 \mathrm{R}-$ profile is consistent with the Beta, Gamma, or Eta variants (B.1.351, P.1, and B.1.525 Pango lineages, respectively), whereas a $484 \mathrm{~K}-/ 484 \mathrm{Q}-/ 452 \mathrm{R}$ + profile is consistent with the Delta variant plus other variants of negligible incidence such as Kappa VOI or $21 \mathrm{C}$ VUM (B.1.427 and B.1.429 Pango lineages, respectively). Other variants include: $20 \mathrm{l} / 501 \mathrm{Y} . \mathrm{V} 1,20 \mathrm{l} / 484 \mathrm{~K}, 20 \mathrm{l} / 484 \mathrm{Q}, 2 \mathrm{Ol} / 452 \mathrm{R}, 20 \mathrm{~B} / 681 \mathrm{H}$ (B.1.1.318), 20A/440K (B.1.619), 20A/477N (B.1.620), 20C/484K, 20C, 20D, 20D/452R, 20D/484K - which were sequenced by the CERBA laboratory.

In the model, screening tests consistent with an Alpha variant are the reference for the RRR. See [13] for details about the methods used. Our analyses were performed on 8,190 samples since 840 samples with missing values for the factors considered in the model were not included. The level of significance is $p<0.05$.

variant), tended to increase in the Hauts-de-France, Îlede-France, Normandie, and Provence-Alpes-Côte d'Azur regions compared with our reference, which were samples lacking the three mutations, consistent with the Alpha variant (Table).

Samples collected outside hospital settings were less likely to originate from Beta/Gamma/Eta variants than from the Alpha variant. Finally, in the Hauts-deFrance and île-de-France regions, we found a temporal increase of Beta/Gamma/Eta variants compared with the Alpha variant. In most regions, we found a temporal increase of 'other' test results compared with the Alpha variant. This trend is more difficult to interpret since these tests may correspond to any variant.

\section{Transmission advantages}

To further quantify the temporal trends, we performed a statistical analysis using a logistic growth model following previous studies $[1,2,9,14]$ and pairwise comparisons between the three main interpretations of the test results, i.e. consistent with infections by Delta, Beta/Gamma/Eta, or Alpha variants. Between 31 May and 21 June, the Delta variant was found to have a mean transmission advantage over the Alpha variant of greater than $79 \%$ (95\% Cl: $52-110 \%)$ in Hautsde-France, Île-de-France, and Normandie (Figure 1A). In these three regions, we also found a significant transmission advantage of the Delta variant over the Beta/Gamma/Eta variants (Figure 1B). Finally, consistently with our previous results [13], we find that Beta/ Gamma/Eta variants have a significant, but smaller, transmission advantage over the Alpha variant in these regions (Figure ${ }_{1} \mathrm{C}$ ). Transmission advantages tend to decrease with time (Supplementary Figure S3), which could be linked to a delay in collecting the cases or a change in selection pressures.

\section{Doubling times}

Using the incidence of Delta variant-positive tests ( 48 4K-/484Q-/452R+; Supplementary Figure S4), we computed epidemic doubling times. When using a 14-day interval, we find low values such as 9.8 days (95 $\mathrm{Cl} \%$ : 6.3-22.6) in île-de-France and 10.5 days (95 Cl\%: 6.921.9) in Hauts-de-France. However, in Île-de-France, this doubling time strongly increases over time and a 21-day interval yields a median doubling time of 26.9 days (Supplementary Figure $\mathrm{S}_{5}$ ). This pattern could be explained by a delay in case reporting or, conversely, by an initial over-representation of superspreading events.

\section{Modelling epidemic scenarios}

Using an epidemiological model of the French epidemic [15], we investigated the potential consequences of our results in the medium term ( 4 months ahead). In Figure 2, we explored four scenarios ( $A, B, C$, and $D)$ that differ by arbitrary levels of vaccine rollout and background contact rate. In scenarios $A$ and $B$, we assume no transmission increase (other than the transmission advantage of the Delta variant) whereas in scenario C and D, we imposed respectively a 10\% from 14 July 2021 and a $20 \%$ from 1 September 2021 increase in background contact rate (compared with June 2021), in order to explore the impact of major summertime loosening of preventive measures and back-to-school effect. In scenarios $A$ and $C$, the vaccine rollout is assumed to result in a $66 \%$ coverage of the whole population on 


\section{FIGURE 1}

Change in relative frequency and estimated transmission advantage between SARS-CoV-2 Alpha, Beta/Gamma/Eta, and Delta variants in Hauts-de-France, Ille-de-France, and Normandie, France, 31 May-21 June 2021

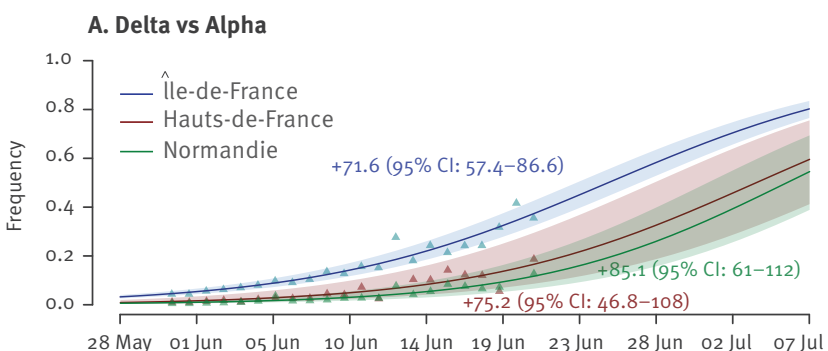

B. Delta vs Beta/Gamma/Eta

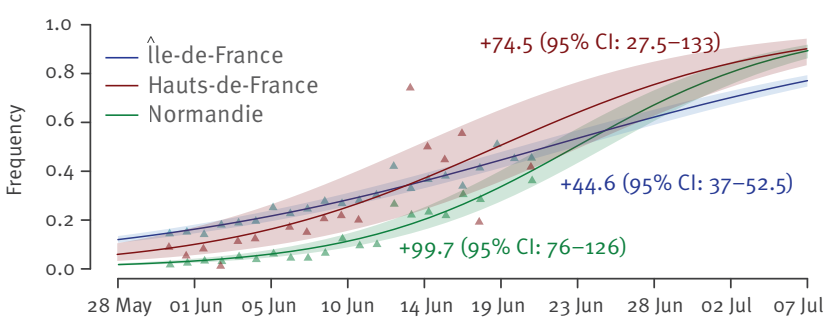

C. Beta/Gamma/Eta vs Alpha

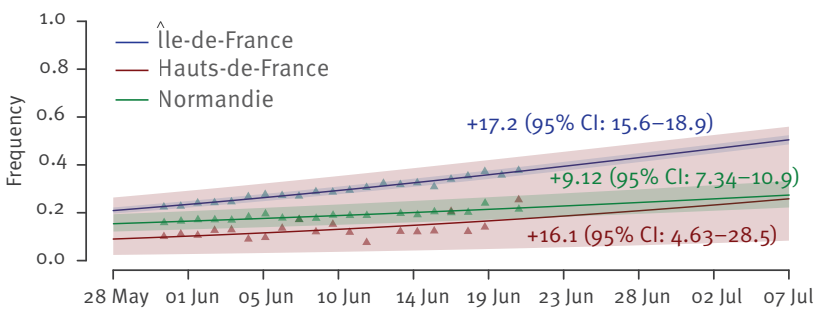

$\mathrm{Cl}$ : confidence interval; SARS-CoV-2: severe acute respiratory syndrome coronavirus 2 .

The triangles indicate the generalised linear model-fitted values and the line indicates the output of the logistic growth model estimation. The shaded area represents the $95 \% \mathrm{Cl}$. The estimated transmission advantages are shown in the panels.

1 September, whereas in scenario $B$, we consider a slower vaccination pace, resulting in ca $61 \%$ vaccination coverage by the end of the summer (see details in the Supplementary Methods). We find that the Delta variant has the potential to initiate an epidemic rebound by the end of the summer that could be amplified by a slowdown in vaccine rollout (Figure 2). Even more, a major increase in background infectious contact rate in September could lead to an intensive care unit (ICU) overload (scenarios C and D). In the absence of any additional mitigation, the residual coronavirus disease (COVID-19) hospital mortality for the second half of 2021 in scenarios A and B remains limited and lies between 10 and $15 \%$ of the current COVID-19 death toll in France. The trend of scenario $C$ or D would, however, call for vigilance and mitigation by the end of the
FIGURE 2

COVIDSIM $^{\text {a }}$ projections of the COVID-19 epidemic for different vaccine rollout and back-to-school effect scenarios, France, June 2021

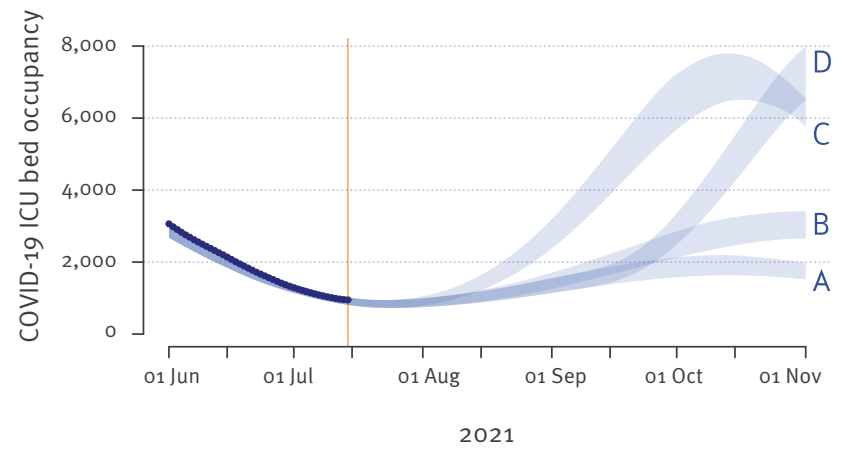

COVID-19: coronavirus disease; ICU: intensive care unit.

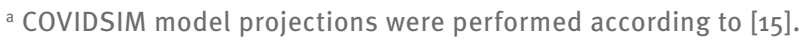

Scenario A: Fast vaccine rollout (66\% of the whole French population is assumed fully vaccinated on 1 September) and constant background contact rate (the transmission increase is solely due to the spread of the Delta variant, assumed to be $70 \%$ more transmissible than the Alpha variant).

Scenario B: Slower vaccine rollout $(-5 \%$ coverage by the end of the summer compared with scenario A) and constant background contact rate.

Scenario C: Fast vaccine rollout and strong summertime loosening of preventive measures $(+10 \%$ increase in background contact rate from 14 July compared with June 2021).

Scenario D: Fast vaccine rollout and strong back-to-school effect $+20 \%$ increase in background contact rate from 1 September compared with June 2021).

The blue stripes represent the current nationwide COVID-19 ICU bed occupancy according to the three scenarios. The width of each stripe corresponds to the range spanned by $95 \%$ of the associated simulations. The turquoise dots are the rolling 7-day average of national COVID-19 ICU bed occupancy. The orange vertical line indicates the day the simulation was performed.

summer if ICU overload is to be avoided and hospital mortality capped.

\section{Ethical statement}

This study has been approved by the Institutional Review Board of the CHU of Montpellier and is registered at ClinicalTrials.gov with identifier NCTo4738331.

\section{Discussion}

Screening for SARS-CoV-2 mutations carried by the Delta variant on samples collected between 31 May and 21 June 2021 indicates that this variant might be spreading rapidly in French regions. According to our estimates, the Delta variant may have already been more frequent than the Alpha variant in Île-de-France by the end of June 2021 . 
There are several potential biases to this analysis, which we attempted to correct. First, since our dataset continually gathers results from local laboratories, there can be delays in data centralisation. To address this, we only analysed the data up to 21 June 2021 and ignored the data collected between 22 and 28 June. Another potential issue relates to the specificity of the test used. Our estimated frequency of the Delta variant was ca $4 \%$ of the positive tests on 8 June. This is consistent with the outcome of national sequencing studies (0.2\% on 11 May 2021 [16]) and the doubling times comparable to those we estimated in the early stages of the Delta variant epidemic in île-de-France (Supplementary Figure S5). Regarding the sampling scheme, the French authorities have announced strict monitoring of the spread of the Delta variant, which could artificially enrich the data with Delta variant-positive tests. We do not know the context in which most tests were performed, except for in a hospital setting and mainly for hospitalised patients. We expect hospital samples to be less impacted by a potential bias related to contact tracing because, aside from nosocomial infections, the contacts of a hospitalised person are unlikely to be hospitalised. Indeed, we find no association between the hospitalisation status and the Delta variant in the multinomial model.

The estimated transmission advantages of $79 \%$ (95\% $\mathrm{Cl}: 52-110 \%)$ the Delta variant over the Alpha variant are in line with that estimated in the UK [7] and from the analysis of the number of variant sequences in the GISAID database [17]. Consistent with our earlier findings from April 2021 [13], we also find that Beta/ Gamma/Eta variants have a transmission advantage over the Alpha variant.

These results have public health implications because they imply that variant Delta could shift epidemic trends. In absence of specific interventions and based on the current vaccine rollout, our model tailored to the French epidemic context suggests that this could cause another COVID-19 wave starting in August 2021. The magnitude and exact timing of this wave would depend on the exact transmission advantage of the variant, the increase in vaccination coverage and a potential loosening of physical distancing and indoor mask-wearing as well as back-to-school effect. This picture could be further complicated by the simultaneous spread of variants Beta, Gamma, and Delta.

\section{Acknowledgements}

We thank Gilles Roullin, Eric Hedbaut and Virginie Dubois from CERBA for their technical assistance, and the ETE team from CNRS, IRD, and University of Montpellier for discussion.

\section{Conflict of interest}

None declared.
Authors' contributions

Bénédicte Roquebert, Sabine Trombert-Paolantoni, Stéphanie Haim-Boukobza, Emmanuel Lecorche, Vincent Foulongne, and Laura Verdurme collected the RT-PCR data. Samuel Alizon performed the statistical analysis. Mircea T. Sofonea performed the mathematical modelling. Samuel Alizon wrote the first version of the article. All the authors contributed to the final version of the manuscript.

\section{References}

1. Davies NG, Abbott S, Barnard RC, Jarvis Cl, Kucharski AJ, Munday JD, et al. Estimated transmissibility and impact of SARS-CoV-2 lineage B.1.1.7 in England. Science. 2021;372(6538):eabg3055. https://doi.org/10.1126/science. abg3055 PMID: 33658326

2. Volz E, Mishra S, Chand M, Barrett JC, Johnson R, Geidelberg $\mathrm{L}$, et al. Assessing transmissibility of SARS-CoV-2 lineage B.1.1.7 in England. Nature. 2021;593(7858):266-9. https://doi. org/10.1038/s41586-021-03470-x PMID: 33767447

3. Faria NR, Mellan TA, Whittaker C, Claro IM, Candido DDS, Mishra S, et al. Genomics and epidemiology of the P.1 SARSCoV-2 lineage in Manaus, Brazil. Science. 2021;372(6544):81521. https://doi.org/10.1126/science.abh2644 PMID: 33853970

4. Tegally H, Wilkinson E, Giovanetti M, Iranzadeh A, Fonseca V, Giandhari J, et al. Detection of a SARS-CoV-2 variant of concern in South Africa. Nature. 2021;592(7854):438-43. https://doi. org/10.1038/s41586-021-03402-9 PMID: 33690265

5. World Health Organization (WHO). Tracking SARSCoV-2 variants. Geneva: WHO. [Accessed: 1 Jul 2021]. Available from: https://www.who.int/en/activities/ tracking-SARS-CoV-2-variants

6. Public Health England (PHE). SARS-CoV-2 variants of concern and variants under investigation in England. Technical briefing 14. London: PHS. [Accessed: 3 Jun 2021]. Available from: https://assets.publishing.service.gov.uk/government/uploads/ system/uploads/attachment_data/file/991343/Variants_of_ Concern_VOC_Technical_Briefing_14.pdf

7. Public Health England (PHE). SARS-CoV-2 variants of concern and variants under investigation - Technical briefing 15; London: PHE; 2021. Available from: https://assets.publishing. service.gov.uk/government/uploads/system/uploads attachment_data/file/993879/Variants_of_Concern_VOC Technical_Briefing_15.pdf

8. Bernal JL, Andrews N, Gower C, Gallagher E, Simmons R, Thelwall $S$, et al. Effectiveness of COVID-19 vaccines against the B.1.617.2 variant. medRxiv. 2021;p.2021.05.22.21257658 https://doi.org/10.1101/2021.05.22.21257658

9. Haim-Boukobza S, Roquebert B, Trombert-Paolantoni S, Lecorche E, Verdurme L, Foulongne V, et al. Detecting rapid spread of SARS-CoV-2 variants, France, January 26-February 16, 2021. Emerg Infect Dis. 2021;27(5):1496-9. https://doi. org/10.3201/eid2705.210397 PMID: 33769253

10. Gaymard A, Bosetti P, Feri A, Destras G, Enouf V, Andronico A, et al. Early assessment of diffusion and possible expansion of SARS-CoV-2 Lineage 20l/501Y.V1 (B.1.1.7, variant of concern 202012/01) in France, January to March 2021. Euro Surveill. 2021;26(9):2100133. https://doi.org/10.2807/1560-7917. ES.2021.26.9.2100133 PMID: 33663644

11. Rambaut A, Holmes EC, O'Toole Á, Hill V, McCrone JT, Ruis $C$, et al. A dynamic nomenclature proposal for SARS-CoV-2 lineages to assist genomic epidemiology. Nat Microbiol. 2020;5(11):1403-7. https://doi.org/10.1038/s41564-020-0770-5 PMID: 32669681

12. Santé Publique France. Analyse de risque sur les variants émergents du SARS-CoV-2 réalisée conjointe-ment par Santé publique France et le CNR des virus des infections respiratoires. Mise à jour du 30/06/2021. [SARS-CoV-2 emerging variant risk analysis jointly realised by French public health national agency (Santé Publique France) and the national reference center (Centre National de Référence) for respiratory infection viruses]. Saint-Maurice cedex: Santé Publique France. [Accessed: 14 Jul 2021]. French. Available from: https://www.santepubliquefrance.fr/media/ files/01-maladies-et-traumatismes/maladies-et-infectionsrespiratoires/infection-a-coronavirus/analyse-de-risque-desvariants-emergents-de-sars-cov-2-30-06-21

13. Roquebert B, Trombert-Paolantoni S, Haim-Boukobza S, Lecorche E, Verdurme L, Foulongne V, et al. The SARS-CoV-2 B.1.351 lineage (VOC $\beta$ ) is outgrowing the B.1.1.7 lineage (VOC a) in some French regions in April 2021. Euro Surveill. 2021;26(23):2100447. https://doi.org/10.2807/1560-7917. ES.2021.26.23.2100447 PMID: 34114541 
14. Chevin LM. On measuring selection in experimental

evolution. Biol Lett. 2011;7(2):210-3. https://doi.org/10.1098/ rsbl.2010.0580 PMID: 20810425

15. Sofonea MT, Reyné B, Elie B, Djidjou-Demasse R, Selinger

C, Michalakis Y, et al. Memory is key in capturing COVID-19 epidemiological dynamics. Epidemics. 2021;35:100459. https://doi.org/10.1016/j.epidem.2021.100459 PMID: 34015676

16. Santé Publique France. Quelle est l'évolution moléculaire des virus SARS-CoV-2 circulant sur le terri-toire? Résultats de l'enquête Flash\#9. [What is the molecular evolution of SARS-CoV-2 viruses circulat-ing in the territory? Flash survey results \# 9]. Saint-Maurice cedex: Santé Publique France. [Accessed: 16 Jun 2021]. French. Available from: https://www. santepubliquefrance.fr/maladies-et-traumatismes/maladieset-infections-respiratoires/infection-a-coronavirus/documents/ enquetes-etudes/quelle-est-l-evolution-moleculaire-des-virussars-cov-2-circulant-sur-le-territoire-resultats-de-l-enqueteflash-9

17. Campbell F, Archer B, Laurenson-Schafer H, Jinnai Y, Konings F, Batra N, et al. Increased transmissibility and global spread of SARS-CoV-2 variants of concern as at June 2021. Euro Surveill. 2021;26(24):2100509. https://doi.org/10.2807/1560-7917.

ES.2021.26.24.2100509 PMID: 34142653 